BULL. AUSTRAL. MATH SOC.

VOL. 33 (1986), 139-144.

\title{
AN ASYPPTOTIC THEOREM FOR ABSTRACT DIFFERENTIAL EQUATIONS
}

\author{
Gaston Mandata n'Guérékata
}

In this paper, we prove the existence of a continuous one-to-one correspondence between bounded solutions of the equation $x^{\prime}(t)=A x(t)+b(t)$ which belong to a certain subclass $L_{+}$of $C_{b}^{O}(R ; X)$ and bounded mild solutions of the equation $x^{\prime}(t)=A x(t)+b(t)+f(t, x(t))$ of the form $u(t)=\phi(t)+\psi(t)$, with $\phi(t) \in L_{+}$and $\lim _{t \rightarrow \infty} \psi(t)=\theta$.

1. Let $X$ be a Banach space with norm $\|\cdot\|$ and $C_{b}^{O}(R ; X)$ the Banach space of continuous and bounded functions: $\mathrm{R} \rightarrow X$ with norm $\|\cdot\|_{0}$. Then consider the following classes of functions:

$L_{+}$is a subclass of functions in $C_{b}^{O}(R ; X)$ with the unique property: $\phi_{1}+\phi_{2} \in L_{+}$for every $\phi_{1}, \phi_{2} \in L_{+}$.

$\mathcal{C}\left(\mathrm{R}^{+} ; X\right)$ is the class of continuous functions $\phi: \mathrm{R}^{+} \rightarrow X$ such that $\lim \phi(t)=\theta$.

$t \rightarrow \infty$

$\stackrel{O}{B}\left(R^{+} ; X\right)$ is the class of functions $u: R^{+} \rightarrow X$ with the property that there exist $\phi \in L_{+}$and $\psi \in \stackrel{O}{C}\left(R^{+} ; X\right)$ such that $u(t)=\phi(t)+\psi(t)$, $t \in \mathrm{R}^{+}$.

Now consider the differential equations in $X$ :

Received 18 June 1985. This research is supported by a visiting professorship at the Université de Montréal in May-June 1985. I gratefully thank Professor Zaidman for the invitation.

Copyright Clearance Centre, Inc. Serial-fee code: 0004-9727/86 $\$ \mathrm{~A} 2.00+0.00$. 


$$
\begin{gathered}
x^{\prime}(t)=A x(t)+b(t), t \in \mathrm{R} \\
x^{\prime}(t)=A x(t)+b(t)+f(t, x(t)), t \in \mathrm{R}
\end{gathered}
$$

and the following assumptions:

(i) the closed linear operator $A$ with domain $D(A)$ dense in $X$ is the infinitesimal generator of a strongly continuous oneparameter group $T(t)$ such that $T(t) x \in L_{+}$for each $x \in X$.

(ii) $b: R \rightarrow X$ is continuous and $b(t) \in L_{+}$.

(iii) $f: \mathrm{R} \times X \rightarrow X$ is continuous in $t$ and $x$ jointly and $\|f(t, x)-f(t, y)\|<L(t)\|x-y\|$, for every $t \in R$ and $x, y \in X$; $\int_{0}^{\infty}\|f(t, \theta)\| d t<\infty$ and $M \int_{0}^{\infty} L(t) d t<1$, where $M=\sup _{t \in \mathbb{R}}\|T(t)\|<\infty$.

Now a mild solution of equation (2) is (by definition) a continuous function $x: \mathrm{R} \rightarrow X$ with the integral representation

$$
x(t)=T(t) x(0)+\int_{0}^{t} T(t-\sigma)(b(\sigma)+f(\sigma, x(\sigma))) d \sigma, t \in R .
$$

(the integral is considered in Bochner's sense). Our theorem below is inspired by a result of Coppel (see [1] proposition 3, p. 35 or [2] theorem 9.5) on almost periodic solutions of exponentially dichotomic equations in $R^{n}$, but it is not a direct generalisation of Coppel's result.

2. THEOREM. Suppose every bounded solution of (1) is in $L_{+}$; then there exists a continuous one-to-one correspondence between bounded solutions of (1) and bounded mild solutions of (2). Moreover every bounded mild solution of (2) restricted to $R^{+}$is in $\stackrel{O}{B}\left(R^{+} ; X\right)$.

Proof. Consider the map $S: C_{b}^{O}(R ; X) \rightarrow C_{b}^{O}(R ; X)$ defined by

$$
S x(t)=\int_{0}^{t} T(t-\sigma) f(\sigma, x(\sigma)) d \sigma, t \in R .
$$

This is well-defined on $C_{b}^{O}(R ; X)$ since 


$$
\begin{aligned}
\|S x(t)\| & \leqslant M \int_{0}^{t}\|f(\sigma, x(\sigma))\| d \sigma \\
& \leqslant M\left(\int_{0}^{t}\|f(\sigma, x(\sigma))-f(\sigma, \theta)\| d \sigma+\int_{0}^{t}\|f(\sigma, \theta)\| d \sigma\right) \\
& \leqslant M\left(\int_{0}^{t} L(\sigma)\|x(\sigma)\| d \sigma+\int_{0}^{t}\|f(\sigma, \theta)\| d \sigma\right) \\
& \leqslant M\left(K \int_{0}^{\infty} L(\sigma) d \sigma+\int_{0}^{\infty}\|f(\sigma, \theta)\| d\right)
\end{aligned}
$$

where $K=\|x\|_{0} \equiv \sup _{t \in \mathbb{R}}\|x(t)\|$. Moreover $S$ is a strict contraction because of the following inequality

$$
\begin{aligned}
\|S x(t)-S y(t)\| & =\left\|\int_{0}^{t} T(t-\sigma)(f(\sigma, x(\sigma))-f(\sigma, y(\sigma))) d \sigma\right\| \\
& \leqslant M \cdot\|x-y\|_{0} \cdot \int_{0}^{\infty} L(\sigma) d \sigma .
\end{aligned}
$$

and the assumption $C \equiv M \int_{0}^{\infty} L(\sigma) d \sigma<1$. Now let $z(t)$ be a bounded solution of $(1)$ and consider the map $F: C_{b}^{O}(R ; X) \rightarrow C_{b}^{O}(R ; X)$ defined by

$$
F x(t)=z(t)+S x(t) .
$$

Then $F$ is also a strict contraction; therefore $F$ possesses a unique fixed point $u(t)$ which satisfies

$$
u(t)=z(t)+S u(t), t \in R,
$$

that is

$$
u(t)=T(t) u(0)+\int_{0}^{t} T(t-\sigma)(b(\sigma)+f(\sigma, u(\sigma))) d \sigma
$$

because $u(0)=z(0)$.

We see $u(t)$ is a mild solution of (2). It is bounded as the sum of two bounded functions.

On another hand, if $u(t)$ is a bounded mild solution (2), it has the representation (3) and if we put $z(t)=T(t) u(0)+\int_{0}^{t} T(t-\sigma) b(\sigma) d \sigma$, which 
implies $z(0)=u(0)$, then $z(t)=u(t)-S u(t)$, and $z(t)$ is a bounded solution of (1).

Now the map $z \rightarrow u$ satisfies the following inequalities

$$
\left\|u_{1}-u_{2}\right\|_{0} \leqslant \frac{1}{1-c}\left\|_{z_{1}}-z_{2}\right\|_{0}
$$

and

$$
\left\|z_{1}-z_{2}\right\|_{0} \leqslant(1+c)\left\|u_{1}-u_{2}\right\|_{0}
$$

Therefore it is a continuous one-to-one correspondence. Finally, let us show that every bounded mild solution $u(t)$ of $(2)$ restricted to $\mathrm{R}^{+}$is in $\stackrel{O}{B}\left(R^{+} ; X\right)$.

We have

$$
u(t)=z(t)+\int_{0}^{t} T(t-\sigma) f(\sigma, u(\sigma)) d \sigma, t \geqslant 0
$$

with $z(t) \in L_{+}$.

Moreover, we can prove the existence in $X$ of the improper integral $\int_{0}^{\infty} T(-\sigma) f(\sigma, u(\sigma)) d \sigma$ because it satisfies the inequality

$$
\left\|\int_{0}^{\infty} T(\sigma) f(\sigma, u(\sigma)) d \sigma\right\| \leqslant M\left(K \int_{0}^{\infty} L(\sigma) d \sigma+\int_{0}^{\infty}\|f(\sigma, \theta)\| d \sigma\right) .
$$

Therefore the function $\mathrm{R} \rightarrow X$ defined by

$$
\int_{0}^{\infty} T(t-\sigma) f(\sigma, u(\sigma)) d \sigma=T(t) \int_{0}^{\infty} T(-\sigma) f(\sigma, u(\sigma)) d \sigma
$$

belongs to $L_{+}$.

Also it is easy to prove that $\int_{t}^{\infty} T(t-\sigma) f(\sigma, u(\sigma)) d \sigma$ exists in $X$ for every $t>0$. And we have

$$
\left\|\int_{t}^{\infty} T(t-\sigma) f(\sigma, u(\sigma)) d \sigma\right\| \leqslant M\left(K \int_{t}^{\infty} L(\sigma) d \sigma+\int_{t}^{\infty}\|f(\sigma, 0)\| d \sigma\right),
$$

which shows $\lim _{t \rightarrow \infty} \int_{t}^{\infty} T(t-\sigma) f(\sigma, u(\sigma)) d \sigma=\theta$. we have proved

$$
u(t)=z(t)+\int_{0}^{\infty} T(t-\sigma) f(\sigma, u(\sigma)) d \sigma-\int_{0}^{\infty} T(t-\sigma) f(\sigma, u(\sigma)) d \sigma \in \stackrel{O}{B}\left(\mathrm{R}^{+} ; X\right) \text {. }
$$


Remark 1. $L_{+}$contains the class of almost automorphic functions: $\mathrm{R} \rightarrow X$ and consequently $\stackrel{O}{B}\left(\mathrm{R}^{+} ; X\right)$ contains the class of asymptotically automorphic functions: $\mathrm{R}^{+} \rightarrow X$ (see [4] and [5] for this concept). In this case, our theorem gives conditions for bounded mild solutions of equations of the form (2) to remain almost automorphic as $t$ goes to infinity, provided bounded solutions of equation (1) are also almost automorphic.

Remark 2. The technique used here can be applied to many problems of asymptotic stability. For example, if we consider equation (1) with $\int_{0}^{\infty}\|b(t)\| d t<\infty$, then every solution of (1) restricted to $R^{+}$is in $\stackrel{O}{B}\left(\mathrm{R}^{+} ; X\right)$. This gives a simple generalisation of Zaidman's result ([6] theorem 1) and is also closed to Lemma 1 ([3]).

\section{References}

[1] W. A. Coppel, Dichotomies in Stabizity Theory, (Springer-Verlag, BerlinHeidelberg-New York, 1978).

[2] A.-M. Fink, Almost Periodic Differential Equations, (Springer-verlag, Berlin-Heidelberg-New York, 1974).

[3] P.A. Mishnaevskii, "The attainment of almost-periodic conditions and the almost-periodicity of solutions of differential equations in a Banach space", Vestnik Moskov. Univ. Mat., 26 (3) (1971), $69-76$.

[4] G.M. N'Guérékata, "Sur les solutions presque automorphes d'équations différentielles abstraites", Ann. Sci. Math. Quebec, V (1) (1981), $69-79$.

[5] G.M. N'Guérékata, "Quelques remarques sur les fonctions asymptotiquement presque automorphes", Ann. Sci. Math. Québec, VII (2) (1983), $185-191$.

[6] S. Zaidman, "Notes on abstract almost-periodicity", Riv. Mat. Univ. Parma, 5 (4) (1979), 837-845. 
[7] S. Zaidman, Almost Periodic Functions in Abstract Spaces, (Pitman Advanced Publishing Program, Boston-London-Melbourne, 1985).

Université de Bangui,

Faculté des sciences,

B.P. 1450 Bangui,

République Centrafricaine. 